\title{
Coronary Chronic Total Occlusion (CTO): A Review
}

\author{
Christian O. Koelbl ${ }^{1, *}$, Zoran S. Nedeljkovic ${ }^{2}$ and Alice K. Jacobs ${ }^{2}$ \\ ${ }^{1}$ Columbia University Division of Cardiology Mount Sinai Heart Institute 4300 Alton Road De Hirsch Meyer Tower Suite \\ 2070 Miami Beach, FL 33140, USA \\ ${ }^{2}$ Section of Cardiology Boston Medical Center 88 East Newton Street Boston, Massachusetts 021 1 8-2393, USA \\ ${ }^{*}$ Correspondence to Christian O. Koelbl: christian.koelbl@msmc.com
}

DOI: $X X X / j . r c m . x x x . x x . x x x$

This is an open access article under the CC BY-NC-ND license (http://creativecommons.org/licenses/by-nc-nd/4.0/)

Coronary artery chronic total occlusions (CTO) are frequently encountered during coronary angiography; however percutaneous recanalization has historically been technically challenging and an important determinant for referral to coronary artery bypass surgery or for medical therapy alone. Recent advances in interventional equipment and innovative approaches to crossing CTO have significantly increased the success rate of percutaneous treatment. Although there is only one relevant randomized control trial (RCT) performed to date, several large, nonrandomized studies have consistently reported improvement in clinical outcomes, including improved survival and relief of angina, when successful percutaneous treatment of CTO was compared with unsuccessful revascularization. These positive observational results have encouraged the initiation of several RCTs which will provide more robust evidence on clinical outcomes of CTO-PCl compared with guideline-directed medical therapy (GDMT) alone.

\section{Keywords}

Chronic total occlusion (CTO); Percutaneous coronary intervention (PCI); Coronary artery disease (CAD);Stable Ischemic Heart Disease (SIHD)

\section{Introduction}

Percutaneous coronary intervention (PCI) of chronic total occlusions (CTO) has been rapidly evolving in recent years. CTO are commonly encountered, occurring in approximately $20 \%$ of all patients referred for coronary angiography (Fefer et al., 2012; Kahn et al., 1993; Christofferson et al., 2005). The prevalence of CTO in patients with known coronary artery disease (CAD) is between 30 to $50 \%$ (Christofferson et al., 2005; Srinivas et al., 2002), and even higher in patients with prior coronary artery bypass graft (CABG) surgery (Fefer et al., 2012; Jeroudi et al., 2013). However, revascularization rates of CTO have historically been low. An attempt at PCI is performed at a rate of only $10 \%$ to $15 \%$ of all patients with CTO (Fefer et al., 2012; Cohen et al., 2003; Grantham et al., 2009).

There are several reasons for the under-representation of CTO as PCI target lesions. Among them may be the paucity of robust Level of Evidence A supporting treatment indications of CTO by
PCI in clinical guidelines (Levine et al., 2011; Windecker et al., 2014). Another reason is the technically challenging nature of the procedure. Technical failure may be due to (1) inability to cross the obstruction with a guidewire, (2) subintimal passage of the guidewire and failure to access the true lumen of the distal vessel, (3) inability to cross the obstruction with a balloon following successful guidewire passage, (4) inability to dilate the lesion secondary to fibrosis or calcification, (5) inability to deliver or expand a stent across the lesion, or (6) perforation of the vessel with a guidewire.

Although there is only one randomized controlled trial (RCT) available to date, a vast and rapidly growing number of observational clinical studies have shown multiple clinical benefits for patients undergoing successful CTO-PCI when performed in experienced centers by skilled operators. Over the last 5 years, available data have evolved from single and multicenter registries and large meta-analyses. In addition, there are currently two well-designed RCTs underway to assess potential clinical benefits of CTO-PCI compared to guideline-directed medical therapy (GDMT). CTOspecific percutaneous techniques and equipment have been developed and refined in recent years, and the procedural risk of CTOPCI has declined steadily in experienced centers. In this article, we provide a critical review of the current literature on CTO-PCI with a focus on clinical outcomes and outline the development of interventional techniques for CTO-PCI.

\section{Definition and Prevalence}

A CTO is defined as an atherosclerotic complete vessel occlusion with Thrombolysis in Myocardial Infarction (TIMI) grade 0 flow within the occluded segment, and an estimated occlusion duration of $\geq 3$ months (Sianos et al., 2008; Stone et al., 2005). Data on the prevalence of CTO are derived from registries of patients undergoing coronary angiography for suspected CAD; thus the overall prevalence in the general (asymptomatic) population is unknown. In patients who are undergoing cardiac catheterization and have a prior diagnosis of $\mathrm{CAD}$, the frequency with which at least one CTO is encountered ranges from $30 \%$ to $50 \%$ (Christofferson et al., 2005; Srinivas et al., 2002). Among patients undergoing PCI in the National Heart, Lung, and Blood Institute Dynamic Registry between 1997 and 1999, CTOs were frequent and more likely encountered in older patients (Cohen et al., 2003). The frequency 
of at least one CTO was $36.5 \%$ in patients $<65$ years, $39.1 \%$ in those $65-79$ years, and $40.7 \%$ in those $\geq 80$ years of age. The right coronary artery was the most commonly affected vessel, followed by the left anterior descending and left circumflex arteries.

A large Canadian multicenter registry reported on 14,439 patients undergoing non-emergent coronary angiography from April 2008 to July 2009 (Fefer et al., 2012). Patients presenting with STsegment elevation myocardial infarction (STEMI) or prior coronary artery bypass surgery (CABG) were excluded from the analysis. Indications for coronary angiography were nearly equally divided between acute coronary syndrome and patients with stable CAD. Among patients with CAD, 1,697 patients (18.4\%) were found to have at least one CTO. This percentage is slightly lower than in other reports, most likely because more moderate CAD was included in the analysis (the study defined CAD by $>50 \%$ stenosis in $\geq 1$ coronary artery, whereas the cutoff in most other studies is $\geq 70 \%$ stenosis). Half of the CTO lesions were located in the right coronary artery.

Jeroudi et al reviewed data on 1,699 patients who underwent coronary angiography between January 2011 and December 2012 in a tertiary Veterans Affairs (VA) hospital in Texas (Jeroudi et al., 2013). In patients with $C A D$ without prior $C A B G$, the prevalence of CTO was $31 \%$ and more than half of all CTO were located in the RCA. In patients with prior CABG, the prevalence was $89 \%$.

\section{Management of CTO}

Despite the frequency with which a CTO is encountered, an attempt at PCI has historically been performed in only $10 \%$ to $15 \%$ of all patients with a CTO (Fefer et al., 2012; Cohen et al., 2003; Grantham et al., 2009). In fact, a CTO has a strong influence on the decision to not perform PCI (Christofferson et al., 2005). In patients with multivessel CAD who were screened for the Bypass Angioplasty Revascularization Investigation (BARI) trial comparing balloon angioplasty to CABG, the presence of a CTO was the most common angiographic characteristic which influenced the decision against enrollment (Bourassa et al., 1995). Similarly, there were four times as many CTO in the parallel Synergy between PCI with TAXUS and Cardiac Surgery (SYNTAX) CABG registry, compared with the number of CTO that were enrolled in the randomized trial (Serruys et al., 2009). In the SYNTAX trial, the presence of a CTO was also the strongest independent predictor of incomplete revascularization after PCI (complete revascularization only achieved in 53\%), but CTO-PCI success rate was only $49 \%$, likely due to limited technical experience at the time (trial enrollment between 2005 and 2007).

In the Canadian registry reflecting the time period from 2008 to 2009 (Fefer et al., 2012), the majority of CTO lesions in the CAD group without prior CABG were left un-revascularized and managed with medical therapy alone (64\% of cases). If revascularization of the CTO lesion was undertaken, it was predominantly performed via CABG (23\% of all CTO patients) and less likely via PCI (10\% had attempted CTO-PCI of which $70 \%$ were successful). Notably, the majority of CTO patients did not have Q-waves on electrocardiogram in the corresponding CTO territory, and > $50 \%$ of CTO patients had preserved left ventricular function, suggesting that myocardial territory supplied by a vessel with a CTO is often viable (due to the presence of collaterals) and could potentially benefit from revascularization.

What is at odds with the low PCI rate for CTO is that almost all CTO territories are ischemic. CTO are chronic by definition, allowing for development of collaterals from other coronary beds. Fractional flow reserve (FFR) is an interventional tool used to assess whether coronary lesions are causing hemodynamic compromise. FFR is very helpful in clinical practice and frequently performed to evaluate the significance of moderate stenoses in order to decide whether PCI is indicated. Interestingly, FFR data have shown that virtually all viable myocardial CTO territories are, in fact, ischemic (Werner et al., 2006). Collaterals to CTO territories often serve to keep the myocardium viable, but provide insufficient blood flow reserve for increased myocardial demands above the resting state. It has been shown that even though baseline FFR values in CTO territories are lower than in patients with significant but not completely occluded coronary lesions, post-PCI FFR will improve to the same absolute level in both CTO and non-CTO lesions, giving patients with successful CTO-PCI an even higher relative benefit than their non-CTO counterparts in terms of FFR improvement (Sachdeva et al., 2014).

In recent years, CTO-PCI has been performed at increased rates in experienced centers. In the VA study by Jeroudi et al (Jeroudi et al., 2013), CTO-PCI was performed at a rate of $30 \%$, and the technical success rate was high $(82 \%)$. However, patients with CTO (compared to CAD patients without CTO) were still more likely to be referred for revascularization with $\mathrm{CABG}$, probably due to a strong association of CTO with multivessel CAD (55\% with 3-vessel CAD in the CTO group, versus $20 \%$ in non-CTO group).

\section{Clinical Guidelines Recommen- dations for CTO-PCl}

The 2011 ACCF/AHA/SCAI guidelines for PCI assign a Class IIa indication with level of evidence B to PCI of a CTO, but do not provide further details on specific indications: "PCI of a CTO in patients with appropriate clinical indications and suitable anatomy is reasonable when performed by operators with appropriate expertise" (Levine et al., 2011). The 2014 European Society of Cardiology/European Association for Cardio-Thoracic Surgery (ESC/EATCS) guidelines for myocardial revascularization also give a Class IIa (B) recommendation, if an ischemia reduction in the CTO territory and/or the relief of angina symptoms can be expected (Windecker et al., 2014). They include the recommendation to preferably perform PCI of a CTO via an antegrade approach, and consider a retrograde approach (IIb; B) after a failed antegrade attempt, or as a primary approach in selected patients.

\section{Clinical Outcomes}

There is currently only one RCT available with reported clinical outcomes of PCI versus GDMT in CTO patients with stable ischemic heart disease (SIHD). However, many observational studies have reported clinical benefits of successful CTO-PCI, including reduced rates of mortality, angina symptoms, major adverse cardiovascular events (MACE), and less need for subsequent CABG (Table. 1). Data are available from the era of balloon angioplasty in the early 1990s to the use of bare metal stents (BMS) 
Table 1. Clinical outcomes of successful CTO-PCI compared to unsuccessful attempts of revascularization in four large studies. Rates of mortality, MACE, angina and need for CABG surgery were reduced after successful CTO-PCI (n/a = data not available).

\begin{tabular}{|c|c|c|c|c|c|c|c|c|c|c|c|}
\hline \multirow{3}{*}{ STUDY } & \multirow{3}{*}{$\begin{array}{c}\text { TOTAL } \\
\text { NO OF } \\
\text { PA- } \\
\text { TIENTS }\end{array}$} & \multirow{3}{*}{$\begin{array}{l}\text { STUDY } \\
\text { DATES }\end{array}$} & \multirow{3}{*}{$\begin{array}{c}\text { MEAN } \\
\text { F/U } \\
\text { PERIOD }\end{array}$} & \multicolumn{2}{|c|}{ MORTALITY } & \multicolumn{2}{|c|}{ MACE } & \multicolumn{2}{|c|}{ CABG } & \multicolumn{2}{|c|}{ ANGINA } \\
\hline & & & & Successfu & 1 Failed & Successf & 1 Failed & Success & 1 Failed & Success & 1 Failed \\
\hline & & & & $\begin{array}{c}\text { CTO- } \\
\text { PCI }\end{array}$ & $\begin{array}{l}\text { CTO- } \\
\text { PCI }\end{array}$ & $\begin{array}{c}\text { CTO- } \\
\text { PCI }\end{array}$ & $\begin{array}{l}\text { CTO- } \\
\text { PCI }\end{array}$ & $\begin{array}{l}\text { CTO- } \\
\text { PCI }\end{array}$ & $\begin{array}{l}\text { CTO- } \\
\text { PCI }\end{array}$ & $\begin{array}{c}\text { CTO- } \\
\text { PCI }\end{array}$ & $\begin{array}{l}\text { CTO- } \\
\text { PCI }\end{array}$ \\
\hline $\begin{array}{l}\text { Christakopoulos } \\
\text { (Werner et al., } \\
\text { 2006) }\end{array}$ & 28,486 & $1990-2014$ & 3.1 years & $6.4 \%$ & $9.5 \%$ & $21.2 \%$ & $35.7 \%$ & $3.3 \%$ & $17.3 \%$ & $25.5 \%$ & $47.5 \%$ \\
\hline $\begin{array}{l}\text { Godino } \\
\text { (Sachdeva et } \\
\text { al., 2014) }\end{array}$ & 1,184 & $1998-2008$ & 4 years & $6.0 \%$ & $12.1 \%$ & $38.7 \%$ & $29.9 \%$ & $3.1 \%$ & $6.0 \%$ & $\mathrm{n} / \mathrm{a}$ & $\mathrm{n} / \mathrm{a}$ \\
\hline $\begin{array}{l}\text { Khan (Chris- } \\
\text { takopoulos et } \\
\text { al., 2015) }\end{array}$ & 12,970 & $1990-2012$ & 3.7 years & $12.5 \%$ & $16.0 \%$ & $22.8 \%$ & $36.6 \%$ & $4.9 \%$ & $20.9 \%$ & $\mathrm{n} / \mathrm{a}$ & $\mathrm{n} / \mathrm{a}$ \\
\hline $\begin{array}{l}\text { Joyal (Godino } \\
\text { et al., 2013) }\end{array}$ & 7,288 & $1979-2006$ & 6 years & $14.3 \%$ & $17.5 \%$ & $28.0 \%$ & $38.7 \%$ & $7.0 \%$ & $26.3 \%$ & $30.2 \%$ & $49.7 \%$ \\
\hline
\end{tabular}

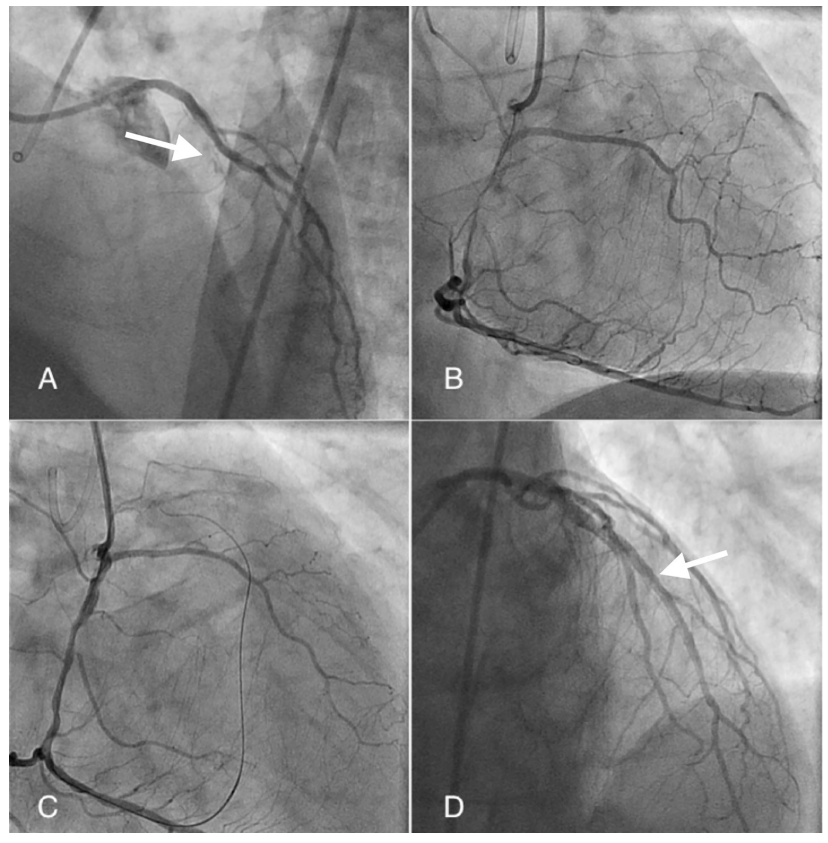

Figure 1. Retrograde guidewire technique of a CTO lesion. (A) Dual catheters. Left coronary artery in LAO cranial view showing CTO of LAD (white arrow). (B) RCA with retrograde filling of LAD via septal collaterals. (C) Coronary guidewire advanced from RCA through septal collateral branch retrograde into LAD. CTO lesion was crossed by retrograde guidewire, which was then advanced into the guide catheter of the left coronary artery and externalized. (D) Angiographic result after successful CTO-PCl with antegrade opacification of LAD (white arrow). [Courtesy of A. David Litvak, MD, Boston Medical Center, Boston, MA].

through the present widespread use of $3^{\text {rd }}$ generation drug-eluting stents (DES). However, data on clinical benefits are almost exclusively derived from retrospective series comparing cohorts of successful PCI with failed CTO-PCI procedures.

A large meta-analysis by Christakopoulos et al compared longterm outcomes of patients with successful CTO-PCI versus un- successful CTO-PCI (Christakopoulos et al., 2015). The authors included 25 studies with publication dates ranging from 1990 to 2014 , encompassing a total of 28,486 patients, or 29,315 attempted CTO-PCI procedures. The average procedural success rate was $71 \%$ (ranging from $51 \%$ to $87 \%$ ) and improved over the observed time period. With a mean follow up of 3.1 years, patients who underwent successful CTO-PCI (versus unsuccessful PCI) had lower mortality rates $(6.4 \%$ versus $9.5 \%$, odds ratio (OR) 0.52 ), less residual angina $(25.5 \%$ versus $47.5 \%$, OR 0.38$)$, a lower stroke risk (2.5\% versus $3.3 \%$, OR 0.72), reduced need for CABG $(3.3 \%$ versus $17.3 \%$, OR 0.18 ), and a lower risk for MACE (21.2\% versus $35.7 \%$, OR 0.59). The benefits of PCI of the CTO lesion were evident for all three revascularization techniques: stenting with DES, stenting with BMS as well as for balloon angioplasty alone. Previous meta-analyses of retrospective series have yielded similar results (Table. 1) (Godino et al., 2013; Khan et al., 2013; Joyal et al., 2010). Other series have also reported improvement in left ventricular function after successful CTO-PCI (Baks et al., 2006; Sirnes et al., 1998).

There are inherent weaknesses and limitations in retrospective data and the meta-analyses derived from them. Non-randomized, observational cohort studies are prone to confounders and selection bias. Patients with unsuccessful CTO-PCI represent a group with higher risk clinical characteristics, including higher rates of previous $\mathrm{CABG}$, more calcified and multivessel disease, and higher degree of LV dysfunction (Christakopoulos et al., 2015; Christopoulos et al., 2014; Michael et al., 2013), which might, at least partially, explain the inferior outcomes in this group. A second possibility is that the failed procedure caused harm to patients (failed with complications) due to periprocedural dissection, coronary perforation, higher rates of contrast induced nephropathy among other adverse events, thereby affecting future clinical outcomes.

Khan et al synthesized data on the immediate complication rates of CTO-PCI and the in-hospital clinical outcomes (Khan et al., 2015). They included 25 studies published between 1991 and 
2013. Their analysis showed that patients with failed CTO-PCI had higher in-hospital mortality (1.4 versus $0.5 \%)$, higher rate of in-hospital MACE (8.9 versus 3.7\%), a slightly higher risk of inhospital myocardial infarction (3.2 versus $2.4 \%$ ), and were more likely to require urgent $\mathrm{CABG}$ (4.0 versus $0.5 \%$ ) when compared to the group of patients with successful CTO-PCI. They also found higher rates of coronary perforations and cardiac tamponade in the failed CTO-PCI group. A major limitation is the lack of knowledge of lesion characteristics in both groups. Differences in parameters such as lesion length, morphology and degree of calcification could confound the differences in procedural outcomes in both groups. While this may account for and offset some of the observed immediate (30-day) benefits of successful CTO-PCI, the absolute risk difference is small and does not account for the sustained benefits in clinical outcomes years after successful CTOPCI. Since the time period of included studies was $>20$ years, the authors were able to assess the temporal trends of clinical outcomes. In both groups of failed and successful CTO-PCI, adverse in-hospital clinical events have decreased over time and are now comparable with the risks of non-CTO PCI.

In accordance with these findings, Patel et al (Patel et al., 2013) found that the overall procedural risks of contemporary CTO-PCI are relatively low. Analyzing 65 studies published between 2000 and 2011 and encompassing 18,061 patients with 18,941 target CTO vessels, the average angiographic success rate was $77 \%$. The pooled complication rates were: $0.2 \%$ for death, $0.1 \%$ risk for emergent $\mathrm{CABG},<0.01 \%$ risk of stroke, $2.5 \%$ for MI (any cardiac biomarker rise), $0.2 \%$ for Q-wave MI, and 3.8\% for contrast induced nephropathy. Coronary perforation was seen in $2.9 \%$, but rarely led to tamponade $(0.3 \%)$. If CTO-PCI was performed via a retrograde approach, the complication rates were comparably low, and the success rate was similar at $79.8 \%$. With regards to temporal trends, the study confirmed that procedural success rates are improving significantly over time, and major complication rates are decreasing significantly from $1.6 \%$ in studies published between 2000 and 2002, to $0.5 \%$ in studies published between 2009 and 2011.

Present-day data of CTO-PCI show a continued increase in procedural success rates in experienced centers (Table. 2). In a multicenter registry of three CTO-experienced centers in the United States, 1,361 patients underwent CTO-PCI from 2006-2011 (Michael et al., 2013). The technical success rate was $85.5 \%$ and procedural success rate was $84.2 \%$, with a major complication rate of $1.8 \%$. Notably, about one third of the patients had prior CABG, and this subset was more likely to have unsuccessful CTO-PCI, as were older patients, and those with target CTO in the right coronary artery. Independent predictors of technical success were the years of CTO-PCI experience of the site, female sex, and no previous $\mathrm{CABG}$. The most current data from CTO-experienced sites in the US show technical success rates $>90 \%$. By strict adherence to a "hybrid approach" algorithm (see "CTO-PCI Techniques" below), experienced CTO operators accomplished a technical success rate of $93.4 \%$ in a population without prior CABG, and $88.1 \%$ in patients with prior CABG (Christopoulos et al., 2014). The overall success rate achieved matches the rates of European and Japanese registries (Galassi et al., 2011; Rathore et al., 2009) (Ta- ble. 2).

The first RCT comparing CTO-PCI to GDMT in patients with SIHD was presented at the America College of Cardiology (ACC) 2017 meeting. The "Drug-Eluting Stent Implantation Versus Optimal Medical Treatment in Patients With Chronic Total Occlusion" ("DECISION-CTO") trial (ClinicalTrials.gov identifier NCT 01078051) was a prospective, multi-center trial with the goal of enrolling at least 1.100 patients at multiple sites in Korea and the Asian-Pacific region. The study was initially designed for superiority of PCI with DES over optimal medical treatment. However, enrollment was slow, and the design was changed to a non-inferiority trial. Even then, the trial had to be stopped early after 6 years due to low recruitment of only 834 patients (the predetermined estimated sample size was 1284). It is primarily the low enrollment that led to the main result of the trial, that PCI is non-inferior but not superior to OMT, when compared towards a combined primary endpoint of mortality, myocardial infarction (MI), stroke and any revascularization at 3 years. However, mortality and stroke both had a trend towards lower rates in the PCI group. Of note, peri-procedural MI (defined as increased level of cardiac markers) was higher in the PCI group. There was also no consistent documentation of symptoms and burden of ischemia prior to randomization, and the cross-over from the medical therapy to the PCI arm was $18.1 \%$. These issues lead to the conclusion that the study was essentially an underpowered trial, with a trend towards improved mortality for CTO-PCI, but leaving it as a hypothesis that will need to be tested in a larger trial. Of note, the PCI success rate was high at $91.1 \%$.

There are currently two other RCTs underway evaluating outcomes of CTO-PCI versus medical therapy. The "Trial to Evaluate the Utilization of Revascularization or Optimal Medical Therapy for the Treatment of Chronic Total Coronary Occlusions" ("EURO CTO", NCT 01760083) is currently being carried out at multiple sites in Europe. The study aims to enroll a sample size of 1.200 patients with stable angina and/or ischemia on non-invasive testing. It will assess whether CTO-PCI with Biolimus-eluting stent plus GDMT is superior to GDMT alone in improving quality of life (QOL) at 12-month follow up, and non-inferior with respect to a composite of all cause death and non-fatal MI at 36 months. The second ongoing RCT is the "Sham-Controlled Intervention to improve QoL in CTOs (“SHINE CTO”, NCT 02784418). Eligible subjects will undergo a blinded randomization to either CTO-PCI or a "sham intervention" without PCI but with conscious sedation, bilateral arterial access, and similar medical therapy including placebo clopidogrel for 6 months. The primary outcome are symptoms measured by the Seattle Angina Questionnaire (SAQ).

\section{CTO-PCI Techniques}

A detailed account of the many different techniques that have been developed and refined to successfully cross and percutaneously treat CTO are beyond the scope of this review. However, we will review how these techniques were developed and how they are applied today. The reader with interest in a more particular description of contemporary CTO-PCI techniques is referred to excellent reviews by Sumitsuji et al (Sumitsuji et al., 2011) and Michael et al (Michael et al., 2012). 
Table 2. Success rates of CTO-PCI by publication.

\begin{tabular}{lccc}
\hline STUDY & TOTAL NO OF PATIENTS & PROCEDURE DATES & SUCCESS RATE \\
\hline Christopoulos (Baks et al., 2006) & 496 & $2012-2013$ & $0.92^{*}$ \\
Michael (Khan et al., 2015) & 1,361 & $2006-2011$ & $0.86^{*}$ \\
Galassi (Patel et al., 2013) & 1,914 & $2008-2010$ & $0.83^{* *}$ \\
Rathore (Michael et al., 2013) & 806 & $2002-2008$ & $0.88^{*}$ \\
Patel (Michael et al., 2013) & 18,061 & $2000-2011$ & $0.77^{* * *}$ \\
Christakopoulos (Werner et al., 2006) & 28,486 & $1990-2014$ & $0.71^{* *}$
\end{tabular}

* technical success rate reported, ${ }^{* *}$ procedural success rate reported, ${ }^{* * *}$ angiographic success rate reported

Over the last decade, there have been a number of technical advances and innovations, largely attributable to the initial work of a group of highly-skilled Japanese operators. Improvements in coronary guidewire design have enhanced the technical success of CTO-PCI, especially with newer hydrophilic guidewires (Corcos et al., 1998), and stiff, specialty guidewires designed for CTO penetration (Saito et al., 2003). Japanese interventional cardiologists have been pioneers of innovation with techniques of simultaneous antegrade and retrograde (via collateral channels) wire manipulation, and controlled antegrade and retrograde subintimal tracking techniques (controlled entering of the subintimal space followed by reentry into the lumen distal to the lesion-CART), to facilitate crossing of CTO lesions (Surmely et al., 2007; Saito, 2008; Surmely et al., 2006). The impact of these techniques has been reported by operators from the Toyohashi Heart Center, Toyohashi, Japan, in a series of 904 consecutive procedures involving PCI for CTO between 2002 and 2008 (Rathore et al., 2009). Impressively, technical success was achieved in $87.5 \%$ of lesions, with a procedural success (defined as technical success without MACE) in $86.2 \%$ of lesions. Increases in the use of different guidewire crossing techniques (parallel wire, retrograde wire, intravascular ultrasound guidance, and CART) occurred each year. In-hospital MACE rates were $1.5 \%$ in the group with successful CTO PCI and slightly higher at $4.4 \%$ in the group with unsuccessful PCI ( $p$ $=0.027$ ). To examine the retrograde wiring technique in the real world, a multicenter registry in Japan collected data of 801 patients who were treated with CTO-PCI at 28 centers across Japan (Tsuchikane et al., 2013). The technical success rate was $85 \%$ and clinical success was achieved in $84 \%$.

The rationale for these various CTO wiring techniques is supported by histopathologic studies of occluded coronary arteries. Sakakura et al. analyzed 95 CTO lesions from autopsy studies (Sakakura et al., 2014). Larger channels with a diameter above $200 \mu \mathrm{m}$ were relatively rare in CTO lesions. However, smaller microchannels $(<200 \mu \mathrm{m})$ are present in CTO lesions (Srivatsa et al., 1997; Katsuragawa et al., 1993), which may explain why tapered wires with a 0.009-inch tip have an advantage over standard 0.014inch wires, as it has been demonstrated in observational series (Saito et al., 2003). Additionally, it can explain why it is often difficult for antegrade wires to pass intraluminal through a CTO, rationalizing subintimal tracking techniques. The study also showed that the proximal cap of a CTO segment frequently has the appearance of an abrupt closure, whereas the distal cap of the occlusion more often has a tapered appearance (79\% tapered appearance in the distal cap vs $48 \%$ in proximal cap). A tapered cap has been shown to be more amenable to PCI due to the ease of wire penetration (Katsuragawa et al., 1993), affirming the utility of retrograde wiring techniques. Fig. 1 illustrates an example of successful retrograde wiring of a CTO lesion and subsequent CTO-PCI. A widely used clinical and angiographic scoring system to predict the probability of successful antegrade guidewire crossing is the Japanese CTO score (J-CTO score). The score was developed by Morino et al. and uses the proximal cap morphology, length of occlusion, coronary calcification and tortuosity, and prior failed attempt to revascularize the CTO as variables (Morino et al., 2011). The score has been validated in other registries, and it has been shown that procedure time increased by approximately 20 minutes for every one-point increase of the J-CTO score (Christopoulos et al., 2015). It is a valuable tool when reviewing the initial angiogram that allows preparation of the optimal strategy of revascularization tailored to each patient and prediction of the need for advanced crossing techniques.

The results of the Japanese experience may be difficult to reproduce in centers where CTO-PCI volume is low, and with operators who lack the CTO procedural volume to become facile with the complex guidewire strategies that have evolved in the CTO community. However, in recent years, experienced centers across North America and Europe have achieved similarly high success rates beyond $90 \%$ for CTO-PCI. The implementation of a unified algorithm on how these techniques can be applied in the percutaneous treatment of CTO has been developed. A so-called "hybrid" crossing algorithm was described by Brilakis et al (Brilakis et al., 2012). It provides a framework for approaching CTO lesions and establishes a hierarchy of different wiring techniques. In this approach, the operator places two guiding catheters, one in the left and one in the right coronary artery (or sometimes in a bypass graft), allowing dual injections and easy transition from antegrade to a retrograde wiring via collateral branches. After a dual injection angiogram is obtained, predefined anatomic features of the CTO lesion can be assessed, e.g. lesion length, presence and anatomy of collateral arteries etc. Next, a "rank order" of different wiring approaches is established based on the lesion parameters. Attempts to cross the lesion are then performed in this order, while monitoring total procedure time, radiation exposure and contrast volume. The performance of this algorithm was also validated in a high-risk group of patients with previous history of CABG, where a procedural success rate of $87.5 \%$ was achieved (Christopoulos et al., 2014). 


\section{Conclusions}

The field of CTO-PCI has evolved rapidly over the past decade, and today experienced centers with high-volume operators are achieving procedural success rates of $90 \%$. There is a high prevalence of CTO in the CAD population, and historically CTOs have been undertreated with regards to percutaneous revascularization. The current evidence on improved outcomes is based almost exclusively on observational data, and thus has its limitations. Nonrandomized data are prone to confounders, and can only be adjusted for known and measured confounders. Meta-analyses combine studies with a heterogeneity of clinical end points and followup periods; there are various CTO definitions in different studies, and the type and adequacy of GDMT is often not assessed, and likely inadequate in older studies, when compared to today's standards. In addition, whether the CTO serves viable myocardium, angina class and degree of ischemia are often not provided. However, significant clinical benefits of successful CTO-PCI have been shown consistently in multiple large series. There is a need for well-designed randomized controlled studies to validate those findings. DECISION CTO, the first RCT, was presented at ACC 2017 and interpreted mostly as a "negative" trial, because it failed to show an edge of CTO-PCI over medical therapy. But the trial was underpowered due to low enrollment and had several shortcomings. A detailed look at the data of DECISION CTO is actually encouraging, since there was a trend towards improved mortality and in particular improved cardiac mortality. It should be noted that the two ongoing RCTs differ from DECISION CTO in that the primary outcome is defined by improvement of symptoms (angina and QoL). Previous randomized trials in patients with SIHD in where CTO is undefined have not been able to show a clear survival benefit for PCI (Boden et al., 2007). However, these trials often included a large percentage of low risk patients, who do not derive a benefit from PCI. It is likely that patients with large ischemic burden in the CTO territory and those who have significant symptoms are benefitting most from revascularization versus medical therapy alone. The currently ongoing ISCHEMIA trial (NCT01471522) is designed to test the hypothesis that patients with stable CAD but large burden of ischemia (in non-CTO territories) will have a mortality benefit with PCI. If ISCHEMIA is positive, it should encourage further CTO trials to evaluate the mortality benefit of CTO-PCI.

CTO-PCI is technically challenging and requires familiarity with advanced techniques and an experienced operator. Evaluating the procedural risk prior to a PCI attempt by detailed study of the CTO lesion characteristics, presence of collaterals, and patient characteristics, is absolutely essential to determine a favorable risk/benefit ratio for this procedure and to provide the best care to each individual patient.

\section{Aknowledgement \\ XXXX}

\section{Conflict of Interest XXXXX}

\section{Reference}

Baks T, Van Geuns R, Duncker DJ, et al. Prediction of left ventricular function after drug-eluting stent implantation for chronic total coronary occlusions. J Am Coll Cardiol. 2006;47(4):721-725.

Boden WE, O'Rourke RA, Teo KK, et al. COURAGE: Optimal Medical Therapy with or without PCI for Stable Coronary Disease. N Engl J Med. 2007;356(15):1503-1516.

Bourassa MG, Roubin GS, Detre KM, et al. Bypass angioplasty revascularization investigation: Patient screening, selection, and recruitment. Am J Cardiol. 1995;75(9 suppl 1):C3-C8.

Brilakis ES, Grantham JA, Rinfret S, et al. A percutaneous treatment algorithm for crossing coronary chronic total occlusions. JACC Cardiovasc Interv. 2012;5(4):367-379.

Christopoulos G, Menon RV, Karmpaliotis D, et al. Application of the "Hybrid Approach" to Chronic Total Occlusions in Patients With Previous Coronary Artery Bypass Graft Surgery (from a Contemporary Multicenter US Registry). Am J Cardiol. 2014;113(12): 1990-1994.

Christakopoulos GE, Christopoulos G, Carlino M, et al. Metaanalysis of Clinical Outcomes of Patients Who Underwent Percutaneous Coronary Interventions for Chronic Total Occlusions. Am J Cardiol. 2015;115(10):1367-1375

Christopoulos G, Wyman RM, Alaswad K, et al. Clinical Utility of the Japan-Chronic Total Occlusion Score in Coronary Chronic Total Occlusion Interventions: Results from a Multicenter Registry. Circ Cardiovasc Interv. 2015;8(7):7632-7640.

Christofferson RD, Lehmann KG, Martin G V, Every N, Caldwell JH, Kapadia SR. Effect of chronic total coronary occlusion on treatment strategy. Am J Cardiol. 2005;95(9):1088-1091.

Cohen $\mathrm{H}$ a., Williams DO, Holmes DR, et al. Impact of age on procedural and 1 -year outcome in percutaneous transluminal coronary angioplasty: A report from the NHLBI Dynamic Registry. Am Heart J. 2003;146(3):513-519.

Corcos T, Favereau X, Guérin $Y$, et al. Recanalization of chronic coronary occlusions using a new hydrophilic guidewire. Cathet Cardiovasc Diagn. 1998;44(1):83-92.

Fefer P, Knudtson ML, Cheema AN, et al. Current perspectives on coronary chronic total occlusions: The Canadian multicenter chronic total occlusions registry. J Am Coll Cardiol. 2012;59(11):991-997.

Galassi AR, Tomasello SD, Reifart N, et al. In-hospital outcomes of percutaneous coronary intervention in patients with chronic total occlusion: insights from the ERCTO (European Registry of Chronic Total Occlusion) registry. Eurolntervention. $2011 ; 7(4): 472-479$.

Godino C, Bassanelli G, Economou Fl, et al. Predictors of cardiac death in patients with coronary chronic total occlusion not revascularized by PCI. Int J Cardiol. 2013;168(2): 1402-1409.

Grantham JA, Marso SP, Spertus J, et al. Chronic Total Occlusion Angioplasty in the United States. JACC Cardiovasc Interv. 2009;2(6):479-486.

Jeroudi OM, Alomar ME, Michael TT, et al. Prevalence and management of coronary chronic total occlusions in a tertiary veterans affairs hospital. Catheter Cardiovasc Interv. 2013;643/October 2013):637-643.

Joyal D, Afilalo J, Rinfret S. Effectiveness of recanalization of chronic total occlusions: A systematic review and meta-analysis. Am Heart J. 2010;160(1):179-187.

Kahn JK. Angiographic suitability for catheter revascularization of total coronary occlusions in patients from a community hospital setting. Am Heart J. 1993; 126(3 I):561-564.

Katsuragawa $M$, Fujiwara $H$, Miyamae $M$, et al. Histologic studies in percutaneous transluminal coronary angioplasty for chronic total occlusion: comparison of tapering and abrupt types of occlusion and short and long occluded segments. J Am Coll Cardiol. 1993;21(3):604-611.

Khan MF, Brilakis ES, Wendel CS, et al. Comparison of procedural complications and in-hospital clinical outcomes between patients 
with successful and failed percutaneous intervention of coronary chronic total occlusions: A Meta-Analysis of Observational Studies. Catheter Cardiovasc Interv. 2015;85(5):781-794.

Khan MF, Wendel CS, Thai HM, et al. Effects of percutaneous revascularization of chronic total occlusions on clinical outcomes: A meta-analysis comparing successful versus failed percutaneous intervention for chronic total occlusion. Catheter Cardiovasc Interv. 2013;82(1):95-107.

Levine GN, Bates ER, Blankenship JC, et al. 2011 ACCF/AHA/SCAI Guideline for Percutaneous Coronary Intervention: Executive Summary: A Report of the American College of Cardiology Foundation/American Heart Association Task Force on Practice Guidelines and the Society for Cardiovascular Angiography a. Circulation. 201 1;124(23):2574-2609.

Michael TT, Papayannis AC, Banerjee S, et al. Subintimal dissection/reentry strategies in coronary chronic total occlusion interventions. Circ Cardiovasc Interv. 2012;5(5):729-738.

Michael TT, Karmpaliotis D, Brilakis ES, et al. Procedural outcomes of revascularization of chronic total occlusion of native coronary arteries (from a multicenter United States registry). Am J Cardiol. 2013;1 12(4):488-492.

Morino Y, Abe M, Morimoto T, et al. Predicting successful guidewire crossing through chronic total occlusion of native coronary lesions within 30 minutes: the J-CTO (Multicenter CTO Registry in Japan) score as a difficulty grading and time assessment tool. JACC Cardiovasc Interv. $2011 ; 4(2): 213-21$.

Michael TT, Karmpaliotis D, Brilakis ES, et al. Impact of prior coronary artery bypass graft surgery on chronic total occlusion revascularisation: insights from a multicentre US registry. Heart. 2013;99(20):1515-1518.

Patel VG, Brayton KM, Tamayo A, et al. Angiographic Success and Procedural Complications in Patients Undergoing Percutaneous Coronary Chronic Total Occlusion Interventions. A Weighted Meta-Analysis of 18,061 Patients From 65 Studies. JACC Cardiovasc Interv. 2013;6(2).

Rathore $S$, Matsuo $H$, Terashima $M$, et al. Procedural and InHospital Outcomes After Percutaneous Coronary Intervention for Chronic Total Occlusions of Coronary Arteries 2002 to 2008. Impact of Novel Guidewire Techniques. JACC Cardiovasc Interv. 2009;2(6):489-497.

Sumitsuji $S$, Inoue $K$, Ochiai $M$, et al. Fundamental wire technique and current standard strategy of percutaneous intervention for chronic total occlusion with histopathological insights. JACC Cardiovasc Interv. 2011;4(9):941-951.

Srivatsa SS, Edwards WD, Boos CM, et al. Histologic Correlates of Angiographic Chronic Total Coronary Artery Occlusions. J Am Coll Cardiol. 1997;29(5):955-963.

Sakakura K, Nakano M, Otsuka F, et al. Comparison of pathology of chronic total occlusion with and without coronary artery bypass graft. Eur Heart J. 2014;35(25):1683-1693.

Sachdeva R, Agrawal M, Flynn SE, et al. The myocardium supplied by a chronic total occlusion is a persistently ischemic zone. Catheter Cardiovasc Interv. 2014;83(1):9-16.

Surmely J-F, Tsuchikane E, Katoh $O$, et al. New concept for CTO recanalization using controlled antegrade and retrograde subintimal tracking: the CART technique. J Invasive Cardiol. 2006; 18(7):334-338.

Saito S, Tanaka S, Hiroe Y, et al. Angioplasty for chronic total occlusion by using tapered-tip guidewires. Catheter Cardiovasc Interv. 2003;59(3):305-311.

Surmely JF, Katoh O, Tsuchikane E, et al. Coronary septal collaterals as an access for the retrograde approach in the percutaneous treatment of coronary chronic total occlusions. Catheter Cardiovasc Interv. 2007;69(6):826-832.

Saito S. Different strategies of retrograde approach in coronary angioplasty for chronic total occlusion. Catheter Cardiovasc Interv. 2008;71(1):8-19.
Sirnes PA, Myreng Y, Mølstad P, Bonariee V, et al. Improvement in left ventricular ejection fraction and wall motion after successful recanalization of chronic coronary occlusions. Eur Heart J. 1998; 19(2):273-281.

Serruys $P$, Morice $M$, Kappetein A. Percutaneous coronary intervention versus coronary-artery bypass grafting for severe coronary artery disease. N Engl J Med. 2009;360(10):961-972.

Sianos G, Barlis P, Di Mario C, et al. European experience with the retrograde approach for the recanalisation of coronary artery chronic total occlusions. A report on behalf of the euroCTO club. Eurolntervention. 2008;4(1):84-92.

Srinivas VS, Brooks MM, Detre KM, et al. Contemporary percutaneous coronary intervention versus balloon angioplasty for multivessel coronary artery disease: A comparison of the National Heart, Lung and Blood Institute Dynamic Registry and the Bypass Angioplasty Revascularization Investigation (BARI) Study. Circulation. 2002;106(13):1627-1633.

Stone GW, Kandzari DE, Mehran R, et al. Percutaneous Recanalization of Chronically Occluded Coronary Arteries. Circulation. 2005:2364-2372.

Tsuchikane $E$, Yamane $M$, Mutoh $M$, et al. Japanese multicenter registry evaluating the retrograde approach for chronic coronary total occlusion. Catheter Cardiovasc Interv. 2013;82(5):654-661.

Werner GS, Surber R, Ferrari $M$, et al. The functional reserve of collaterals supplying long-term chronic total coronary occlusions in patients without prior myocardial infarction. Eur Heart J. $2006 ; 27(20): 2406-2412$.

Windecker S, Kolh P, Alfonso F, et al. 2014 ESC/EACTS Guidelines on myocardial revascularization: The Task Force on Myocardial Revascularization of the European Society of Cardiology (ESC) and the European Association for Cardio-Thoracic Surgery (EACTS). Eur Heart J. 2014;35(37):2541-2619. 